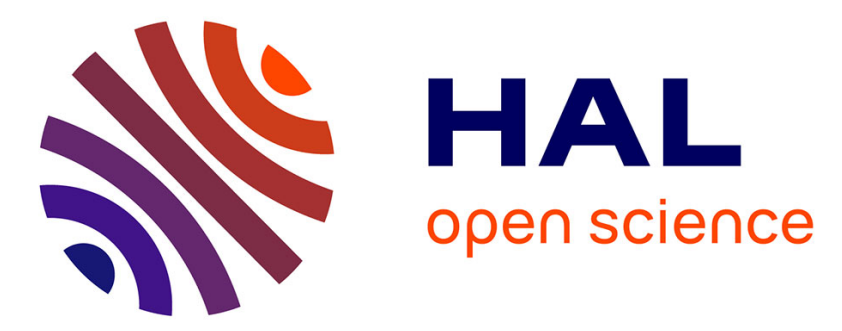

\title{
First experimental programs with the free electron laser
} G. Margaritondo, C. Coluzza, J.-L. Staehli, E. Tuncel, J. Mckinley, R. Albridge, A. Barnes, A. Ueda, X. Yang, N. Tolk

\section{To cite this version:}

G. Margaritondo, C. Coluzza, J.-L. Staehli, E. Tuncel, J. Mckinley, et al.. First experimental programs with the free electron laser. Journal de Physique IV Proceedings, 1994, 04 (C9), pp.C9-357-C9-364. 10.1051/jp4:1994960 . jpa-00253523

\section{HAL Id: jpa-00253523 https://hal.science/jpa-00253523}

Submitted on 1 Jan 1994

HAL is a multi-disciplinary open access archive for the deposit and dissemination of scientific research documents, whether they are published or not. The documents may come from teaching and research institutions in France or abroad, or from public or private research centers.
L'archive ouverte pluridisciplinaire HAL, est destinée au dépôt et à la diffusion de documents scientifiques de niveau recherche, publiés ou non, émanant des établissements d'enseignement et de recherche français ou étrangers, des laboratoires publics ou privés. 


\title{
First experimental programs with the free electron laser
}

G. Margaritondo, C. Coluzza, J.-L. Staehli, E. Tuncel, J.T. McKinley*, R.G. Albridge*, A.V. Barnes*, A. Ueda*, X. Yang* and N.H. Tolk*

Institut de Physique Appliquée, Ecole Polytechnique Fédérale de Lausanne, 1015 Lausanne, Switzerland * Department of Physics and Astronomy, Vanderbilt University, Nashville, Tennessee 37235, U.S.A.

\begin{abstract}
Free electron lasers (FEL's) are not new instruments: for a long time, they have produced highintensity photon beams, primarily in the infrared. They can be, therefore, excellent complementary facilities to synchrotron radiation, whose primary spectral domains are the ultraviolet and the $x$ rays. The use of free electron lasers for experiments, however, has been quite limited; this situation is rapidly changing, with programs being put in operation at Santa Barbara, at Vanderbilt University in Nashville, at the LURE laboratory in Orsay, at the FOM in Holland and elsewhere. We present here our practical experience with one of the first programs using the Vanderbilt FEL -which also constitutes at present one of the largest materials research programs with an FEL. Concrete results will be presented in two areas: two-photon absorption and internal photoemission measurements of interface energy barriers (the so-called FELIPE technique). The discussion will analyze the specific problems and requirements of this class of experiments. The main point, however, is the practical evidence that the FEL can sustain long-term research programs very much like synchrotron facilities. The future possibilities are also briefly discussed, in particular as far as the possible extension of FEL's to the x-rays is concerned.
\end{abstract}

\section{THE ADVENT OF FEL'S IN PRACTICAL RESEARCH PROGRAMS}

For many years, synchrotron sources have provided photon beams of high quality for experiments, primarily in the ultraviolet and $x$-rays domains. Free electron lasers have been developed and proposed as the natural counterparts of synchrotrons for the infrared. Much technical work has been done, and the FEL's have become quite sophisticated. But their use for experiments has been quite limited, so much that some questions have been raised about the possibility of practicality of using these machines.

Recently, the implementation of the first steady and long-term experimental programs based on FEL's have eliminated these questions. Programs of this kind are not very many, but their results are quite significant; we note, for example, the steady progress made at Santa Barbara, and the beginning operation of the FOM facility in the Netherlands and of the LURE FEL in Orsay. The Vanderbilt FEL center has been at the forefront of these developments, both in medical research and in materials science.

We will briefly describe some of the resent accomplishments in materials science research by the Vanderbilt-EPF Lausanne collaboration in Nashville. We believe that these practical results are the best way to illustrate, on one hand, the power of an FEL, and on the other hand the possibility to use it in practice.

\section{TWO-PHOTON SPECTROSCOPY WITH THE FEL}

The Vanderbilt FEL enabled us (see Tuncel et al., Ref. 1) to eliminate an important and long-standing gap in the knowledge $2-13$ of nonlinear optical properties: the two-photon absorption of germanium. No data 
was available on the two-photon absorption at the indirect gap:2,3 the last measurements of this type, 5 performed in 1976 with conventional lasers, did not reach the direct gap; the fundamental interest of their results did nevertheless stimulate much theoretical work. $2,13,14$

Two-photon spectroscopy has been for many years an important component of solid-state research, because of its selection-rule complementarity with respect to conventional optical spectroscopy, and because of the many interesting and nontrivial features of two-photon absorption. ${ }^{2-14}$ Originally treated by Göppert-Mayer, 12 this spectroscopy could be practically implemented only after the invention of lasers, 4 and became one of the basic tools of solid-state physics.

The Vanderbilt FEL provided us with the necessary intensity and tuning range to finally extend this technique to the direct and indirect gaps of germanium. This instrument is an upgraded version of the Stanford University Mark III FEL. 15 The electron beam is produced by a $45-\mathrm{MeV}$ radio frequency accelerator, operating at a frequency of $2.856 \mathrm{GHz}$. The source is tunable over the $2-10 \mathrm{~mm}$ wavelength range (first harmonic, down to $1 \mathrm{~mm}$ in third harmonic) with high output power and brightness. Pulses with $6 \mathrm{~ms}$ duration, $360 \mathrm{~mJ}$ energy and $11 \mathrm{~W}$ average power (repetition rate $30 \mathrm{~Hz}$ ) have been reliably demonstrated in tests conducted at the wavelength of $4.8 \mathrm{~mm}$.

Figures $1 \mathrm{a}$ and $1 \mathrm{~b}$ show the two-photon induced photoconductivity spectrum in the energy region of the direct and indirect Ge gaps. We note immediately the presence of the indirect-gap two-photon absorption threshold in Fig. 1b. The direct and indirect absorption edges cannot be seen on the same scale, because the latter is three orders of magnitude smaller. 1

The photoconductivity for Fig. 1 was measured after biasing the Ge $\mathrm{p}^{+}-\mathrm{i}-\mathrm{n}^{+}$structure at $-300 \mathrm{~V}$ to fully deplete the intrinsic region; a transimpedance amplifier converted photoconductivity into a voltage change, $\Delta V$, which in turn was measured by a boxcar integrator. A reference FEL intensity signal $V_{\mathrm{I}}$ was provided by a Au-doped Ge detector which, due to Au impurity levels within the Ge gap, has broad spectral sensitivity dominated by single photon absorptions. The reference signal was required to eliminate the effects of FEL intensity fluctuations (from 5 to $10 \%$ between macropulses). After testing the quadratic dependence of the absorption on the FEL intensity (see Figs. 2, to be discussed later), we plotted the data as $\Delta V N_{\mathrm{r}}^{2}$ vs double the photon energy, $2 \mathrm{h \omega}$.

From the threshold of Fig. 1a, we estimated a direct gap width $E_{\mathrm{g}} \mathrm{d}=0.87 \pm 0.01 \mathrm{eV}$. This evaluation was further tested by using Mahan's excitonic theory,14 with $E_{\mathrm{g}} \mathrm{d}$ as an adjustable parameter, obtaining again $0.87 \mathrm{eV}$. This value is also reasonable considering the published value 16,17 and the estimated sample temperature: a gap of $0.87 \mathrm{eV}$ corresponds to approximately $125 \mathrm{~K}$.

The $E_{\mathrm{g}} \mathrm{d}$ value from this data analysis was then used to analyze the indirect gap data. Specifically, we assumed that the difference between the gaps is independent of temperature (the published dependence 16,17 would in fact be negligible within our level of accuracy). From the indirect gap width 16,17 at $77 \mathrm{~K}$ of $0.737 \mathrm{eV}$ and the direct-gap decrease of $19 \mathrm{meV}$ from 77-125 K, we derived an indirect gap width $E_{\mathrm{g}} \mathrm{i}=0.718 \pm 0.01 \mathrm{eV}$ at $125 \mathrm{~K}$. The position is shown by the left-side arrow labeled as $E_{\mathrm{g}}{ }^{\mathrm{i}}$ in Fig. $1 \mathrm{~b}$.

The two-photon indirect-gap threshold of Fig. $1 \mathrm{~b}$ is clearly shifted with respect to $E_{\mathrm{g}} \mathbf{i}$, even considering the experimental uncertainty. As shown by the arrow labeled as $E_{\mathrm{g}} \mathbf{i}+\mathrm{LO}$, the shift is consistent with a process assisted by the emission of a $30.4 \mathrm{meV}$ LO $\left(\mathrm{L}_{6}{ }^{-}\right)$phonon. 17 The two-photon indirect threshold is therefore, as predicted by Bassani and Hassan in 1972, primarily an LO-assisted process, as opposed to the one-photon mechanism which can be assisted by several different types of phonons. 2,16

The fits of Figs. $2 a$ and $2 b$ show that the near-threshold intensity dependence is essentially quadratic. This is reasonable, since the deviations from such a behavior ${ }^{5}$ are expected to be small for our experimental conditions, due to the weakness of the two-photon absorption coefficient, to the relatively moderate thickness of the specimen, and to the relatively small incident intensity. 
Note that the quadratic dependence implies a carrier lifetime longer than the transit time. This is consistent with the very high sample purity and with the low carrier concentration produced by two-photon absorption (estimated to never exceed $2 \times 10^{9} \mathrm{~cm}^{-3}$ for the present data). There is also consistency with several other points: saturation effects were not observed, the estimated free-carrier absorption is negligible with respect to two-photon absorption, and the laser intensity does not appreciably change over the sample's depth.
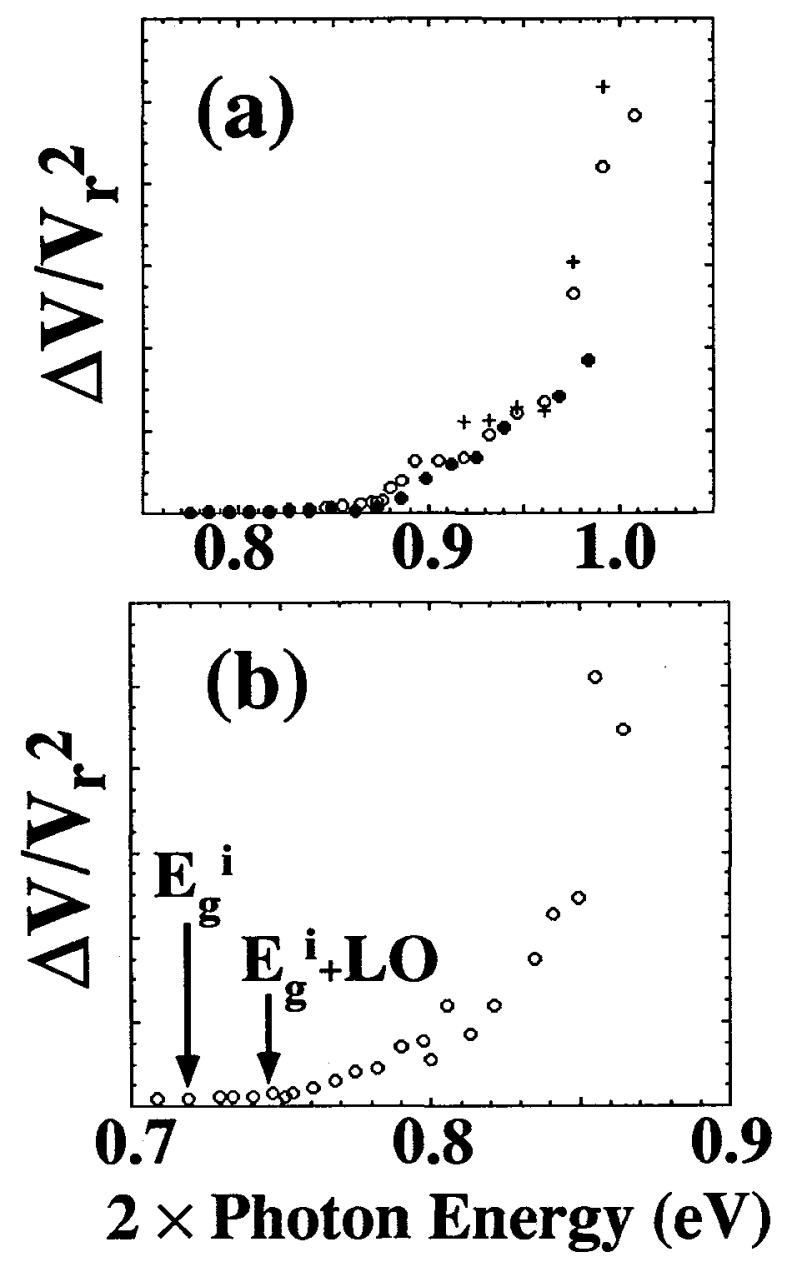

Fig. 1 - (a) Two-photon-absorption induced photoconductivity signal (normalized to the square of the intensity reference signal $V_{\mathrm{r}}$ ), as a function of double the incident photon energy. We note that direct-gap threshold, corresponding to the gap width of $0.87 \mathrm{eV}$ at $125 \mathrm{~K}$. (b) Similar data for the indirect gap. The vertical arrows mark the indirect gap width, $\mathrm{Eg}_{\mathrm{g}} \mathrm{i}$, and the corresponding threshold for LO-phonon assisted processes as predicted by Bassani-Hassan theory. ${ }^{2}$ Data from Tuncel et al., Ref. 1 .

The photoconductivity measurements were complemented by photoluminescence measurements, which did reveal the direct gap but whose signal was too weak to reaveal the indirect gap. The photoconductivity measurements revealed a decrease of the absorption coefficient by approximately three orders of magnitude on going from the direct to the indirect gap spectral region. All of these results finally eliminated a gap in two-photon absorption spectroscopy, concerning one of the most important semiconductors and which affected this field for amost two decades. 


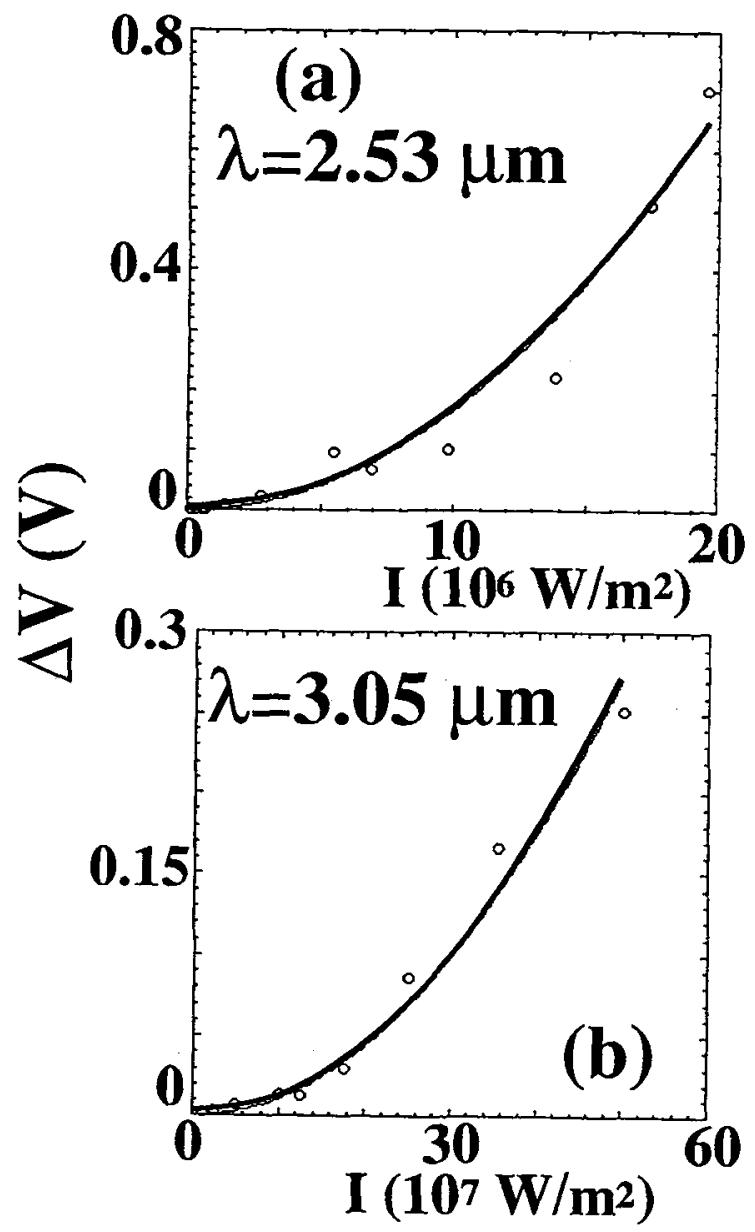

Fig. 2 - (a) Dependence on the incident intensity of the relative magnitude of the photoconductivity induced signal. The data are for a photon energy of $0.490 \mathrm{eV}$ (above the two-photon direct gap threshold), and again for an estimated temperature of $125 \mathrm{~K}$. (b) Similar data for a photon energy of $0.407 \mathrm{eV}$, between the direct and indirect gap thresholds. The solid lines indicate in both cases a quadratic dependence. Data again from Tuncel et al., Ref. 1. 


\section{INTERFACE BARRIER MEASUREMENTS: THE FELIPE TECHNIQUE}

Measuring interface energy barriers is one of the most crucial problems in today's condensed matter science and technology. 18,19 Conventional approaches to these measuremens are affected by several limitations, affecting their accuracy, reliability and domain of applicability. Recently, the vanderbil FEL has been used for a novel approach in a collaborative effort, led by he EPF-Lausanne scientists Carlo Coluzza and JeanLouis Staehli in cooperarion with Norman Tolk, James McKinley and co-workers of Vanderbilt. 20
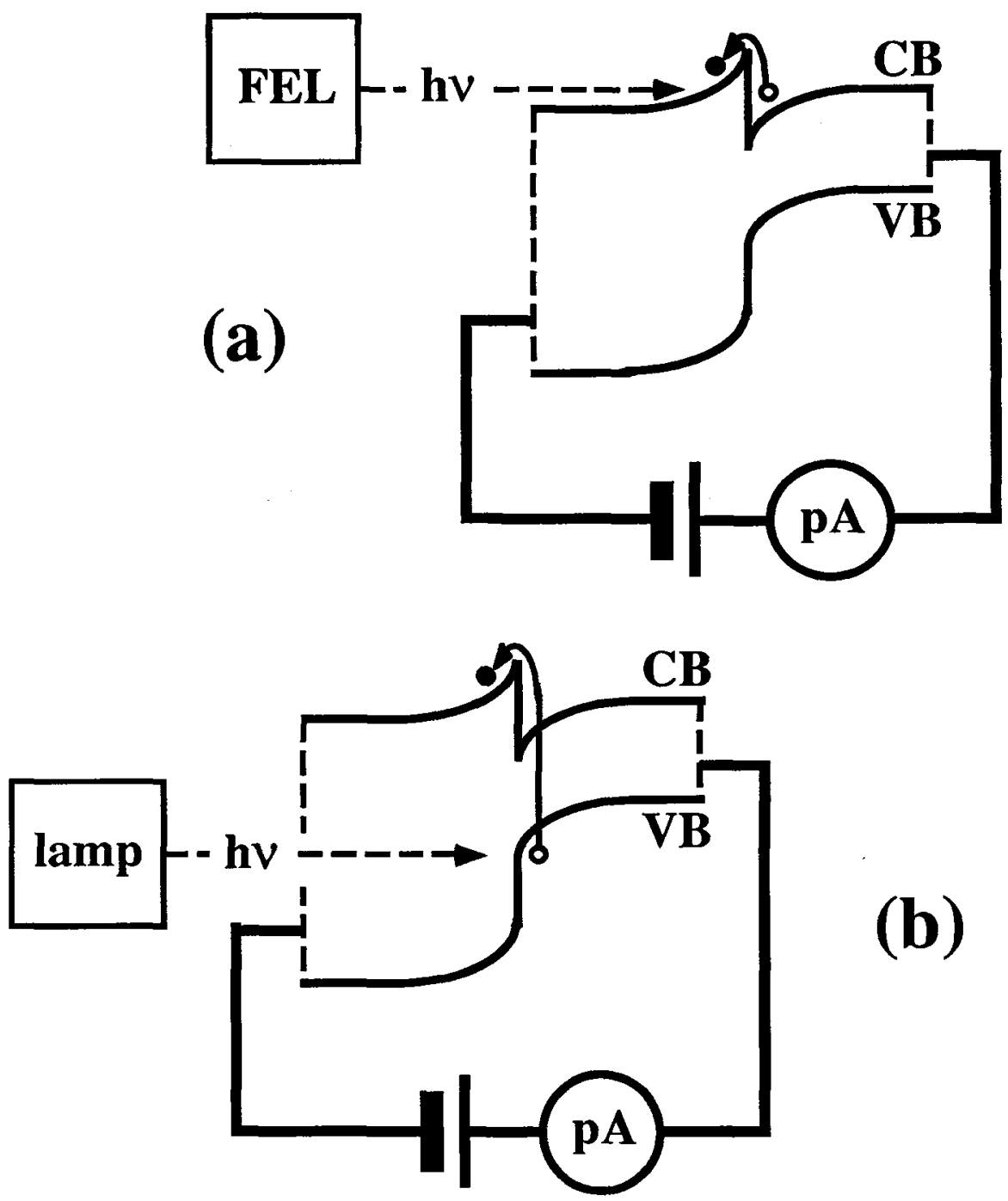

Fig. 3 - Top: FEL internal photoemission (FELIPE) measurements of a semiconductor interface energy barrier: 20,22 when the photons have energy larger than the barrier, one measures a photocurrent in the external circuit. Bottom: similar but more indirect measurements with a conventional source. 
The motivations for initiating this program were compelling: the infrared radiation emitted 15 by the Vanderbilt FEL offered the opportunity to solve two major problems in the physics of interfaces. First, improving the accuracy in measuring interface energy barriers to the level required to test the current theories. 20 Second, to perform such tests on buried interfaces rather than on those formed by thin overlayers.

The FEL approach to measure interface energy barriers is based on the method of internal photoemission, 20 illustrated in Fig. 3 and code-named "FELIPE" (FEL Internal PhotoEmission"). The top part of the figure illustrates an experiment with the FEL, in which photons are used to pump electrons across the energy barrier caused by a heterojunction band discontinuity. The barrier height can be simply and directly derived from the spectral position of the threshold.

The bottom part of the figure shows an alternate method based on a conventional photon source, in which the threshold corresponds to the sum of the discontinuity plus the local gap width. This method is currently used 20,21 to provide information on the local optical properties of the junction's materials, and also to cross-check the results of the more direct FEL measurements, thereby enhancing their reliability -- which is a serious problem in other interface barrier measurements.

Figure 4 shows the first experimental data obtained with this method, 7 which enabled the scientists from our Centre de Spectromicroscopie to measure with high accuracy the discontinuity of a GaAs-AlGaAs heterojunction. Figure 5 shows more recent data of even higher quality. 21 With data of this level, one can easily, directly and reliably assess the barrier height, with an accuracy that changes somewhat from experiment to experiment depending on the specific experimental conditions -- but falls always in the range of a very few meV.

The FELIPE experiments have been refined to the point of becoming almost routine -- the major problem being the development of the required structures for measuring new kinds of interfaces thereby extending its domain of applications.

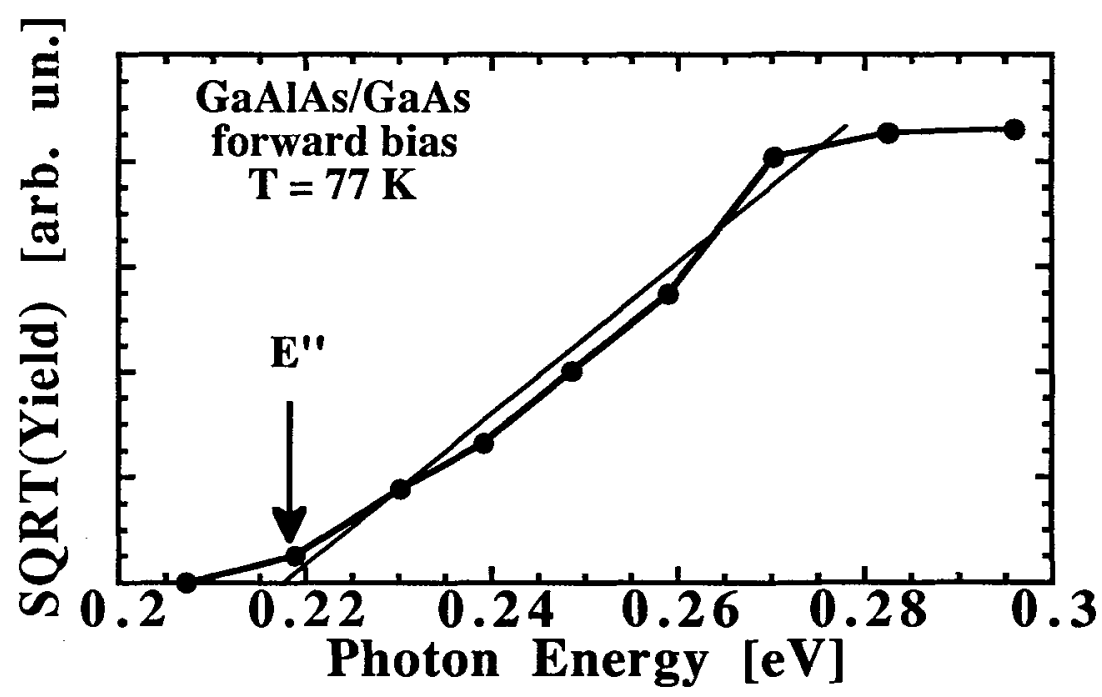

Fig. 4 - FEL internal photoemission data from a GaAs-AlGaAs interface: the conduction band discontinuity is directly and reliably derived from the spectral threshold, E". Data from Coluzza et al., Ref. 20. 


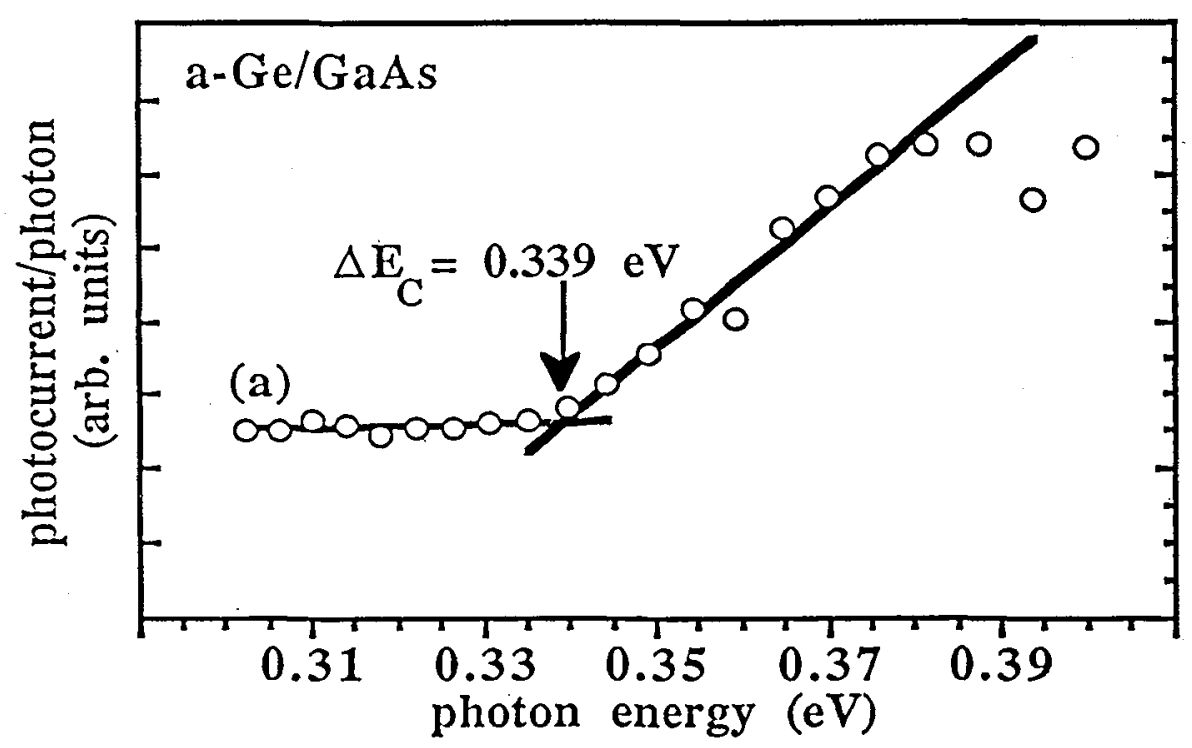

Fig. 5 - FEL internal photoemission data from a heterojunction of amorphous Ge and GaAs. Data from McKinley et al., Ref. 21 .

\section{FUTURE OUTLOOK}

The previously discussed practical experiments are just the tip of the iceberg as far as the potential research applications of FEL'd are concerned. In the immediate future, the Lausanne-Vanderbilt collaboration is planning to extend the FEL to several new types of condensed matter experiments:

- Two-photon photoemission studies of excited states.

- The study of surface photovoltage and charging effects, in particular the "brute force" unpinning of the Fermi level.

- Two-photon spectroscopy of quantum wells and other microstructures.

We are also considering the proposed plans 22,23 for a conversion of the SLAC accelerator into a soft or hard x-ray, ultrashort-pulsed FEL source. A number of interesting applications have been proposed:

- Two-photon spectroscopy of deep core levels. 23

- Fluorescence experiments in the frequency domain, extending a technique that has been implemented at the Frascati laboratory by Gratton, De Stasio and their coworkers. 23

- Novel desorption spectroscopy experiments, for example those based on multiparticle detection and/or $\mathrm{x}$-ray excitation. 23

- Photoemission spectromicroscopy, reaching the natural limitations of this technique.23,24

All of these examples, once again, only give a preliminary idea of the research opportunities: the sky is the limit, together with the creativity of interested scientists. W note that the grandiose plans for the conversion of the SLAC accelerator are not the only possible approach to an ultraviolet or $x$-ray FEL. Several other schemes are being considered, such as an extension of the preliminary FERMI project (Free Electron Radiation and Matching Instrumentation) recently proposed for the ELETTRA laboratory in Trieste. 


\section{ACKNOWLEDGMENTS}

The experiments described in this article habe been performed in collaboration with J.-P. Baudat, $H$. Berger, F. Gozzo, H. Jotterand, C. A. Brau, G. S. Edwards, M. H. Mendenhall, and all the collaborators of the Vanderbilt University Free Electron Laser. Our FEL work was supported by the Office of Naval Research under contracts N00014-87-C-0146, N00014-91-C-0109, and Grant N00014-91-J-4040, by the Fonds National Suisse de la Recherche Scientifique and by a special international cooperation grant from the Ecole Polytechnique Fédérale de Lausanne.

\section{REFERENCES}

[1] Tuncel E., Staehli J. L., Coluzza C., Margaritondo G., McKinley J. T., Yang X. and Tolk, N. H. Phys. Rev. Letters 70 (1993) 4146-4150.

[2] Bassani F. and Hassan A. R., Nuovo Cim. 7B (1972) 313-320.

[3] Bassani F. and Pastori-Parravicini G., Electronic States and Optical Transitions in Solids (Pregamon Press, Oxford, 1975); Hassan A. R., Nuovo Cim. 13B (1973) 19-25.

[4] For an extensive review of the experiments in this field, see: Nathan V., Guenther A. H. and Mitra S. S., J. Opt. Soc. Am. B 2 (1985) 294-310.

[5] Gibson A. F., Hatch C. B., Maggs P. N. D., Tilley D. R. and Walker A. C., J. Phys. C 9 (1976) 3259-3265.

[6] Zubov B. V., Kulevskii L. A.,x Makarov L. A., Murina T. M. and Prokhorov A. M., Zh. Eksp. Teor. Fiz. Pis'ma Red. 9 (1969) 221-225 [Sov. Phys. JETP Lett. 9 (1969) 130-134].

[7] Wenzel R. G., Arnold G. P. and Greiner N. R., Appl. Opt. 12 (1973) 2245-2255.

[8] Reintjes J. F. and McGroddy J. C., Phys. Rev. Letters 30 (1973) 901-905.

[9] Ashkinadze B. M., Pyshkin S. L., Ryvkin S. M. and Yaroshetskii I. D., Fiz. Tekh. Poluprov. 1 (1967) 1017-1025 [Sov. Phys. Semicond. 1 (1968) 850-860].

[10] Catalano I. M., Cingolani A. and Minafra A., Opt. Commun. 11 (1974) 254-258.

[11] Lee C. C. and Fan H. Y., Phys. Rev. B 9 (1974) 3502-3510.

[12] Göppert-Mayer M., Ann. Phys. (Liepzig) 9 (1936) 273-310.

[13] Bryant G. W., Phys. Rev. B 22 (1980) 1992-2010.

[14] Mahan G. D., Phys. Rev. 170 (1968) 825-850.

[15] Tolk N. H., Brau C. A., Edwards G. S., Margaritondo G. and McKinley J. T., Short-Wavelength Radiation Sources, (SPIE Proceedings Series, Bellingham, Washington, 1991) pp. 7-11.

[16] McLean T. P., Progress in Semiconductors Vol. 5, ed. Gibson A. F. (Heywood, London 1960) pp. 53-70.

[17] Landolt- Börnstein Numerical Data and Functional Relationships in Science and Technology Gp III Vol 17a, eds. Cardona M., Harbeke G., Rössler A. and Madelung P. (Springer, Berlin 1982) pp. 87-91.

[18] Margaritondo G., Introduction to Synchrotron Radiation (Oxford, New York 1988); Czaja W., Selected Experiments in Condensed Matter Physics with Synchrotron Radiation (Birkhäuser, Basel 1991); Coluzza C., Sanjinés R. and Margaritondo G., Photoemission: from the Past to the Future (EPFL, Lausanne 1992).

[19] Margaritondo G., J. Vac. Sci. Technol. B11 (1993) 1362-1370, and the references therein.

[20] Coluzza C., Tuncel E., Staehli J.-L., Baudat P. A., Margaritondo G., McKinley J. T., Ueda A., Barnes A. V., Albridge R. G., Tolk N. H., Martin D., Morier-Genoud F., Dupuy C., Rudra A. and Ilegems M., Phys. Rev. B46 (1992) 12834-12838.

[21] McKinley J. T. et al., unpublished.

[22] Scientific Applications of Short Wavelength Coherent Light Sources, eds. Spicer W. and Winick H.

(SLAC, Stanford 1993).

[23] Margaritondo G., Cerrina F., Tolk N. H. and De Stasio G., in Ref. 22 pp. 65-75.

[24] Margaritondo G. and Cerrina F., Nucl. Instr. Meth. A291 (1990) 26-35. 